\title{
Laser à colorant à cavité en anneau, pompé par flash et de grande qualité spatiale
}

\author{
J. P. Boquillon et O. Bazemlal \\ Laboratoire de Spectronomie Moléculaire $(*)$, 6, bd Gabriel, 21000 Dijon, France
}

(Reçu le 19 janvier 1981, révisé le 24 février 1981, accepté le 4 mars 1981)

\begin{abstract}
Résumé. - Nous avons réalisé un laser à colorant pompé par flash dont nous décrivons les performances dans cet article. Grâce à une attention particulière portée à la conception de l'alimentation électrique et des courants de préionisation, ainsi qu'au calcul et à la réalisation de la cavité interférométrique en anneau à 4 miroirs et filtrage spatial, nous obtenons une bonne puissance crête (quelques dizaines de $\mathrm{kW}$ en $500 \mathrm{~ns}$ ) et une très bonne qualité géométrique du faisceau. Ce laser pourra être utilisé en amplificateur à grand gain par injection d'un faisceau monofréquence continu de quelques $\mathrm{mW}$.
\end{abstract}

\begin{abstract}
We realized a flashlamp-pumped dye laser whose results are described in this paper. Owing to a special attention drawn to the design of the electrical supply and of the simmer currents in the same way as the calculation and the realization of the interferometric ring cavity with four mirrors and spatial filtering, we obtain a good peak power (some tens of $\mathrm{kW}$, in $500 \mathrm{~ns}$ ) and a very good spatial beam quality. This laser will be used a high gain amplifier by injection of a monofrequency $\mathrm{CW}$ beam of some $\mathrm{mW}$.
\end{abstract}

1. Introduction. - De nombreuses expériences de spectroscopie à haute résolution par effets non linéaires (CARS ou Raman Stimulé par exemple), nécessitent des sources spectrales puissantes et de bonne qualité spectrale, pouvant être accordées sur une large étendue de longueurs d'onde.

Il faut donc arriver à combiner les qualités de puissance des lasers impulsionnels et finesse spectrale des lasers continus. La solution la plus intéressante semble être l'amplification d'un laser continu à colorant, soit dans des cellules amplificatrices excitées par un laser impulsionnel puissant (YAG doublé par exemple) [1], soit par injection du faisceau laser continu dans la cavité d'un laser à colorant pompé par flashs [2, 3].

Dans ce dernier but, nous avons réalisé un laser à colorant impulsionnel, en attachant une grande importance à la conception de l'alimentation électrique, de façon à conserver un bon rendement et surtout à la cavité interférométrique, afin d'obtenir une bonne qualité spectrale et spatiale sans placer trop d'éléments à l'intérieur de la cavité, qui introduiraient des pertes importantes et compliqueraient le balayage en longueur d'onde.

Nous allons décrire la construction d'un tel laser, facile à mettre en œuvre et de coût modéré, pouvant donc être réalisé en plusieurs exemplaires. La cavité

(*) ERA CNRS no 599. dotée d'un asservissement de longueur nous permettra de réaliser facilement une amplification à grand gain, sans élargissement de la raie continue injectée.

2. Montage expérimental. - 2.1 TÊTE LASER. Pour obtenir des impulsions lumineuses relativement courtes, de l'ordre de la microseconde, il est nécessaire d'utiliser des flashs linéaires ou coaxiaux à faible inductance. Les flashs coaxiaux présentent des avantages pour la facilité du montage et l'homogénéité du pompage optique du colorant, mais leur durée de vie reste limitée à quelques centaines de milliers de tirs, du fait qu'il n'est pas possible de leur appliquer une tension de préionisation. Comme d'autres auteurs [4], nous avons choisi des flashs linéaires dont la durée de vie sera 10 à 20 fois supérieure, tout en essayant de garder une grande facilité de montage et un bon couplage de la cavité réflectrice de pompage.

Pour cela, nous utilisons une cavité classique bielliptique (Fig. 1) équipée de deux flashs ILC type 4D6. Le tube de colorant placé au foyer commun des deux ellipses est un tube en Pyrex dépoli intérieurement, de diamètre égal à celui des flashs soit $4 \mathrm{~mm}$, de diamètre extérieur $8 \mathrm{~mm}$ et de longueur $15 \mathrm{~cm}$.

La cavité réflectrice bielliptique est obtenue suivant une technique de soufflage d'un tube en Pyrex de $60 \mathrm{~mm}$ de diamètre, préalablement amolli et placé dans un moule en graphite [5], chauffé sur une rampe à gaz. Ce moule a été usiné à la forme désirée par fraisage 


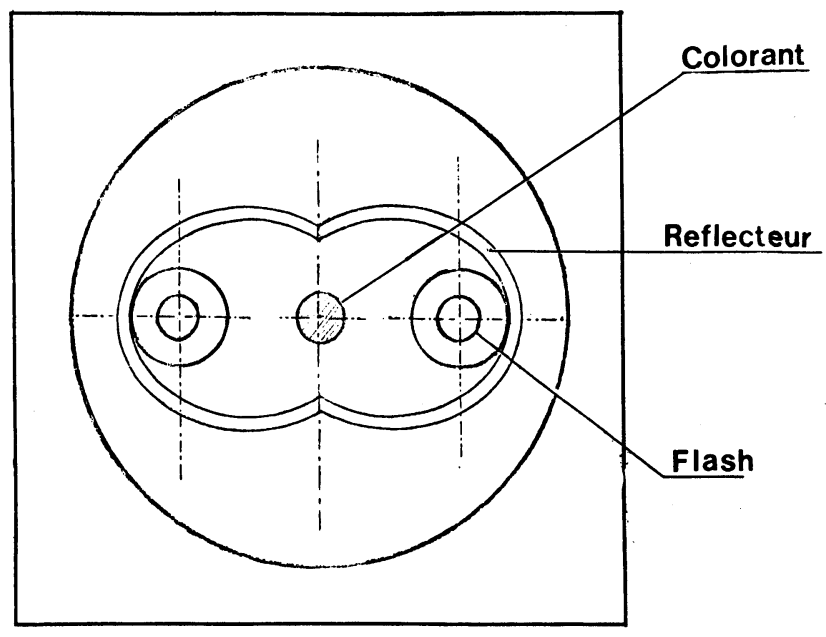

Fig. 1. - Coupe de la tête laser.

[Cross-section of the laser head.]

de deux blocs parallélépipédiques de $150 \mathrm{~mm}$ de longueur, réassemblés ensuite. Le tube en verre ainsi soufflé est coupé à la longueur voulue puis recuit. $\mathrm{La}$ face extérieure du tube est alors métallisée sous vide par évaporation d'une couche d'argent ou d'aluminium, protégée par vaporisation d'un vernis. Nous obtenons ainsi un très bon pouvoir réflecteur, bien supérieur à celui obtenu par polissage d'une pièce métallique massive et surtout très durable, puisqu'il n'y a pas oxydation au contact de l'eau de refroidissement ni attaque par les rayons UV des flashs. $\mathrm{La}$ réalisation d'un tel réflecteur est facile et peu onéreuse, il peut être reproduit en un grand nombre d'exemplaires.
Le refroidissement des flashs est assuré par une circulation d'eau désionisée, en circuit fermé avec stabilisation de température à $0,1^{\circ} \mathrm{C}$ près, par passage dans un échangeur eau-eau.

Les fenêtres de la tête laser, assurant l'étanchéité du circuit de colorant, sont en silice fondue, polies à $\lambda / 10$. Elles font un angle de 2 à 3 degrés avec l'axe optique et entre elles, afin d'éviter de former des cavités parasites bien que les faces aient reçu un traitement anti-reflet.

La circulation de colorant a lieu en circuit fermé avec des matériaux résistants aux solvants (verre, téflon et acier inoxydable). La température du colorant est également stabilisée par passage dans un échangeur placé dans le circuit qui comporte en série, un réservoir de deux litres, une pompe à entraînement magnétique, un filtre millipore et un amortisseur de vibrations. Le débit est d'environ un litre par minute, permettant de renouveler complètement la solution de colorant présente dans la tête laser entre deux tirs, même à une cadence de $20 \mathrm{~Hz}$.

2.2 Circuit Électrique. - L'alimentation électrique est réalisée de façon classique par la décharge d'un condensateur de $0,3 \mu \mathrm{F}$ (Maxwell série SS $35 \mathrm{kV}$ ) à faible inductance $(20 \mathrm{nH})$, à travers un éclateur fabriqué au laboratoire, commandé par un circuit de déclenchement délivrant des impulsions de $25 \mathrm{kV}$ environ à partir d'une suite de circuits logiques. Le condensateur est chargé par une alimentation haute tension avec charge à courant constant (Candela type HVD $1000 \mathrm{~A}$ ) qui permet de travailler à cadence élevée du fait de sa puissance élevée $(1000 \mathrm{~J} / \mathrm{s})$ et de son bon rendement.

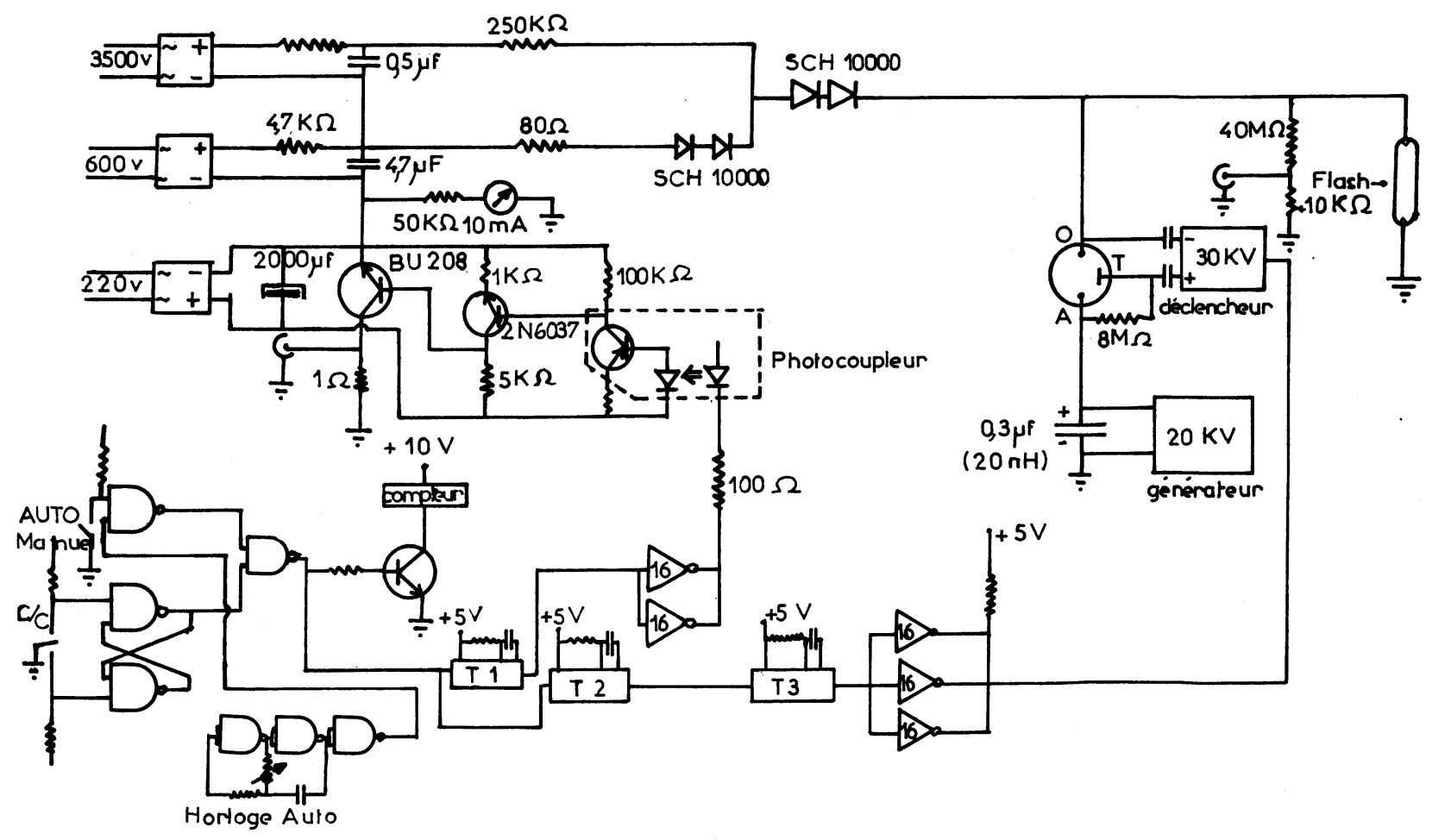

Fig. 2. - Schéma de l'alimentation électrique des flashs. [Schematic diagram of flashlamp electrical circuit.] 
Pour augmenter la durée de vie des flashs et diminuer la durée de l'impulsion lumineuse, donc augmenter l'efficacité du pompage, il est intéressant de diminuer l'impédance du tube flash avant la décharge et pour cela on utilise un circuit de préionisation dit simmer. Deux solutions sont possibles : soit utiliser une ionisation de quelques $\mathrm{kV}$ avec un courant de 10 à $30 \mathrm{~mA}$ [6]. Ceci présente l'inconvénient de ne pas abaisser suffisamment l'impédance des flashs, donc diminue le rendement. La seconde solution consiste à utiliser un fort courant de préionisation voisin de $1 \mathrm{~A}$ [7] qui remplit bien les tubes flash, donc favorise la décharge principale. L'inconvénient majeur de cette technique est qu'elle consomme beaucoup d'énergie, ce qui complique le refroidissement et surtout diminue la durée de vie du colorant illuminé en permanence. Nous avons pensé combiner les avantages des deux méthodes en superposant deux courants de préionisation [8]. Le premier est un courant continu de $12 \mathrm{~mA}$ sous $4000 \mathrm{~V}$ auquel nous ajoutons un courant de $5 \mathrm{~A}$ sous $600 \mathrm{~V}$ provenant de la décharge d'un condensateur auxiliaire de $10 \mu \mathrm{F}$, ceci juste avant la décharge principale, la synchronisation étant assurée par des circuits logiques. Ce courant de préionisation qui dure seulement quelques dizaines de microsecondes, n'échauffe donc pas le colorant tout en remplissant bien les tubes flash.

Dans un proche avenir, nous allons remplacer l'éclateur par un thyratron EGG CX 1538 au fonctionnement plus fiable et au déclenchement plus précis qui permettra de synchroniser deux ou plusieurs lasers identiques.

2.3 CaVité INTERFÉROMÉTRIQUe. - Dans le but de réaliser une amplification par injection, il est plus intéressant de travailler avec des ondes progressives qu'avec des ondes stationnaires [3]. Le pompage du colorant est alors plus homogène, ce qui permet d'extraire plus d'énergie de la cavité puisqu'on utilise un volume plus grand de milieu actif, tout en n'excitant qu'un nombre limité de modes. Un seul élément passif intra-cavité est utilisé (filtre interférentiel) pour affiner la raie émise.

Le schéma optique est donné sur la figure 3, avec

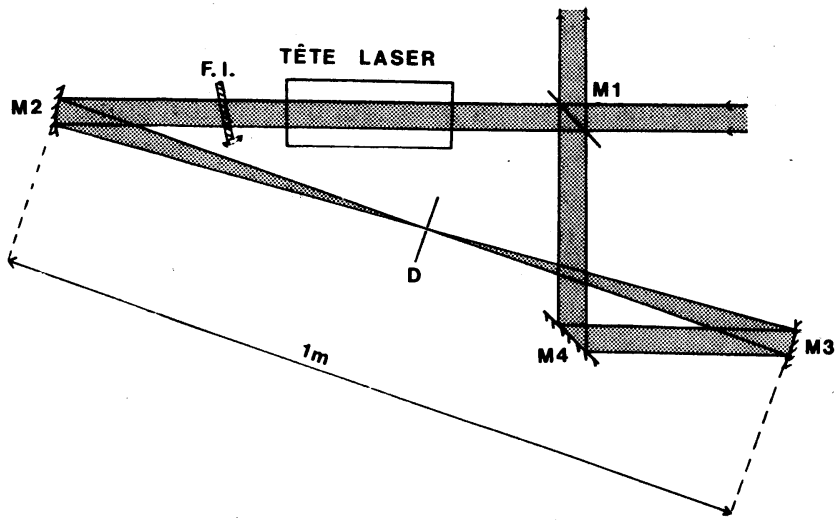

Fig. 3. - Schéma de la cavité laser en anneau.

[Schematic diagram of laser ring cavity.]

REVUE DE PHYSIQUE APPLIQUÉE. - T. 16, No 6, JUIN 1981 une cavité à quátre miroirs [3]. $\mathrm{M}_{1}$ et $\mathrm{M}_{4}$ sont des miroirs plans de coefficients de réflexion respectifs $50 \%$ et $100 \% . M_{2}$ et $M_{3}$ sont des miroirs $R=100 \%$, concaves de rayon de courbure $1 \mathrm{~m}$, placés en position confocale. Ces deux miroirs étant utilisés en dehors de l'axe, il y a apparition d'astigmatisme qui doit être compensé en plaçant $\mathbf{M}_{3}$ en dehors du plan $\mathbf{M}_{1} \mathbf{M}_{2} \mathbf{M}_{4}$ de façon que les plans $M_{1} M_{2} M_{3}$ et $\mathbf{M}_{2} \mathbf{M}_{3} \mathbf{M}_{4}$ soient orthogonaux. Les angles d'incidence sur $\mathbf{M}_{2}$ et $\mathbf{M}_{3}$ sont identiques puisque les rayons de courbure de ces miroirs sont égaux. Un diaphragme $D$ de diamètre $500 \mu \mathrm{m}$ placé au milieu de $\mathrm{M}_{2} \mathrm{M}_{3}$ joue le rôle de filtre spatial, ce qui augmente considérablement la qualité optique et diminue d'autant la divergence du faisceau laser émis.

Aucun sens de circulation des ondes dans la cavité n'étant privilégié, nous avons deux ondes se propageant en sens contraire, ce qui donne en sortie deux faisceaux orthogonaux d'égale intensité. Il est possible d'utiliser une cavité à 3 miroirs [9] avec $M_{1}$ et $\mathbf{M}_{3}$ miroirs plans et $\mathbf{M}_{2}$ miroir concave. La cavité se trouve alors dans un plan mais, pour compenser l'astigmatisme introduit par $\mathbf{M}_{2}$, il faut placer la tête laser à incidence brewstérienne [10] et imposer la valeur de l'angle d'inclinaison de $\mathbf{M}_{2}$. De plus, ce type de cavité ne permet pas l'utilisation d'un filtrage spatial.

La longueur totale de la cavité que nous utilisons est voisine de $3 \mathrm{~m}$ et les différents angles d'inclinaison varient de 10 à $20^{\circ}$. Nous avons également placé entre la tête laser et le miroir $\mathbf{M}_{2}$, un filtre interférentiel $(T=90 \%, \Delta \lambda=20 \AA)$ centré sur $6100 \AA$ et qui, par rotation, permet de faire varier la longueur d'onde d'émission du laser. Ce filtre est utilisé pour la Rhodamine $6 \mathrm{G}$, mais d'autres filtres permettent de travailler avec des colorants différents.

3. Résultats expérimentaux. - Nous avons testé la cavité laser, d'abord en ondes stationnaires avec deux miroirs éloignés de $70 \mathrm{~cm}$, un miroir $R=100 \%$ ayant un rayon de courbure de $3 \mathrm{~m}$ et un miroir $R=50 \%$ plan. Le seuil laser est de $20 \mathrm{~J}$ et la durée

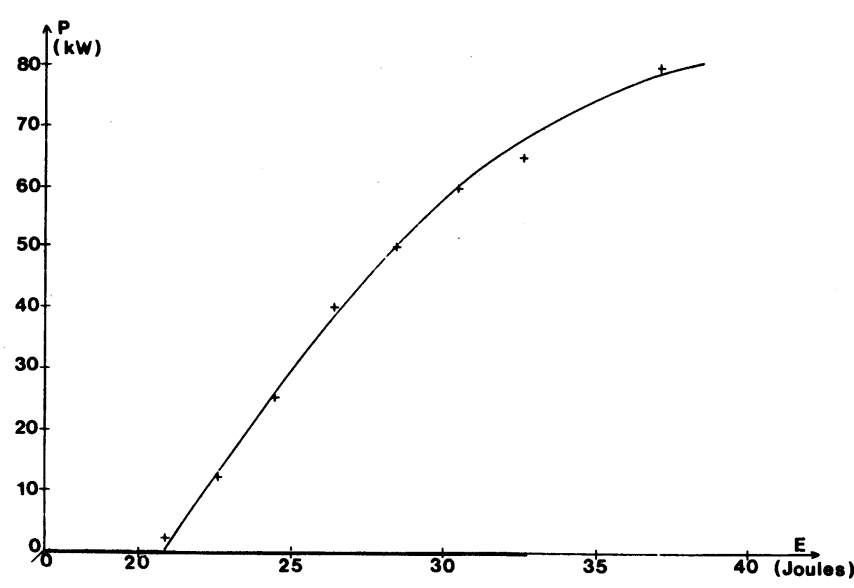

Fig. 4. - Puissance de sortie du laser en fonction de l'énergie d'entrée.

[Laser output power versus total energy input to flashlamps.] 
de l'impulsion émise est d'environ $800 \mathrm{~ns}$. La divergence du faisceau est assez importante : quelques mrad. Nous avons ensuite testé la cavité en ondes progressives avec les 4 miroirs. Dans ce cas, le seuil laser est de $22 \mathrm{~J}$ et nous avons tracé la puissance de sortie en fonction de l'énergie d'entrée (Fig. 4) pour une cavité sans filtre spatial ni filtre interférentiel, la puissance de sortie étant la somme des faisceaux émergents. Il est à noter que dans ce cas, la durée de l'impulsion est deux fois plus courte que dans le cas de la cavité à deux miroirs, les pertes étant moins élevées dans cette configuration.

Nous allons étudier maintenant les résultats obtenus dans le cas qui nous intéresse le plus, de la cavité à quatre miroirs.

3.1 INFLUENCE DES COURANTS SIMMER. - Sans préionisation, le seuil laser est de $37 \mathrm{~J}$, avec le simmer continu seul, il tombe à $27 \mathrm{~J}$ et avec les deux préionisations il est de $22 \mathrm{~J}$, ce qui correspond à une tension de charge du condensateur égale à $12,5 \mathrm{kV}$. L'avantage de. la préionisation est surtout sensible au voisinage du seuil où nous travaillerons, afin d'éviter la naissance de modes inutiles. La figure 5 montre deux tirs réalisés

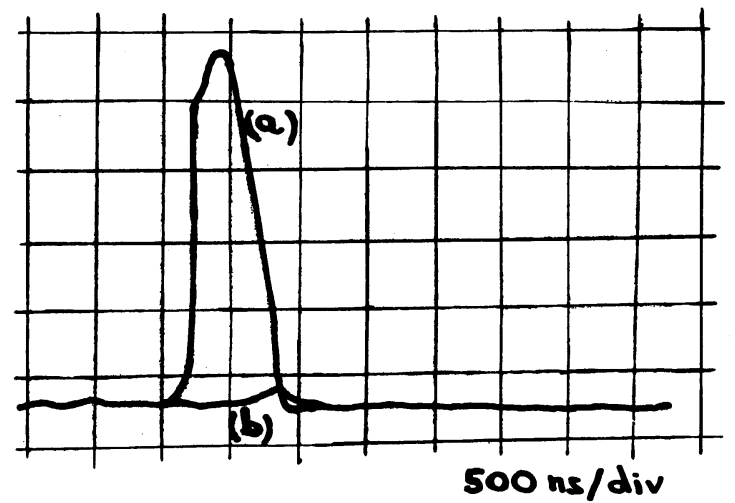

Fig. 5. - a) Impulsion laser avec préionisations près du seuil b) Impulsion laser sans préionisation $\}(27 \mathrm{~J})$.

[a) Laser pulse with simmers enhancement

b) Laser pulse without simmers near threshold $(27 \mathrm{~J})$.]

avec la même tension de charge, soit $13,8 \mathrm{kV}$ avec et sans simmer et sur la figure 6 avec les deux, puis avec le simmer continu seul. De plus, lorsque les préionisations sont présentes, la stabilité en puissance augmente fortement du fait que les flashs travaillent dans des conditions identiques d'un tir à l'autre; c'est ce que l'on peut voir sur la figure 7 où nous avons superposition de 100 tirs successifs.

3.2 Caractéristiques du faisceau laser. - La puissance de sortie est diminuée d'un peu moins de $50 \%$ lorsque l'on place les filtres spatial et interférentiel et le seuil d'émission augmente de quelques joules. Nous obtenons typiquement une puissance de sortie de $20 \mathrm{~kW}$ avec une durée d'impulsion de $400 \mathrm{~ns}$ et une énergie de décharge de $30 \mathrm{~J}$, ceci au maximum d'émission du laser, soit vers $5950 \AA$ en utilisant une solution

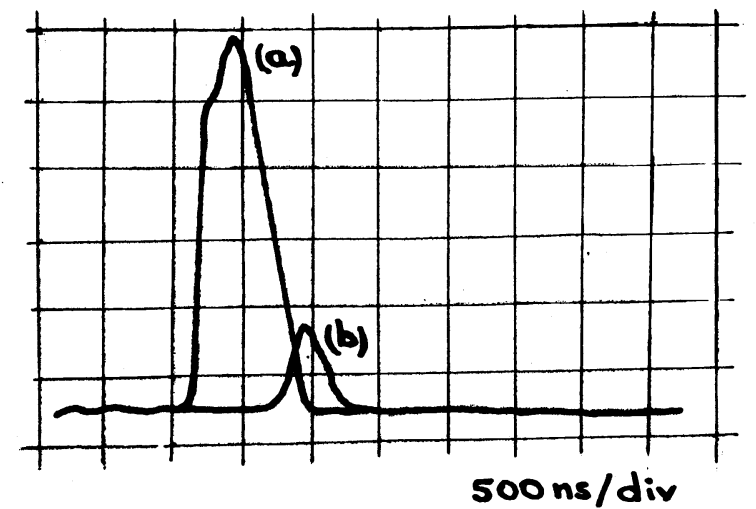

Fig. 6. - a) Impulsion laser avec préionisations b) Impulsion laser avec la préionisation
continue seule $(E=27 \mathrm{~J})$.

$\left.\begin{array}{l}\text { [a) Laser pulse with simmers } \\ \text { b) Laser pulse with } \mathrm{CW} \text { simmer alone }\end{array}\right\}(E=27 \mathrm{~J})$.]

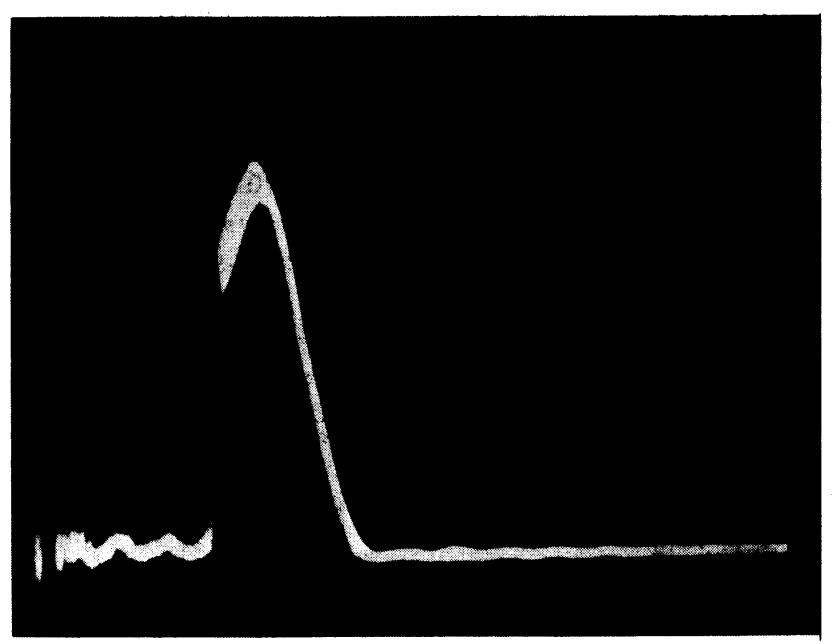

Fig. 7. - Superposition de 100 tirs successifs. [Superposition of 100 successive laser shots.]

de Rhodamine 6G diluée dans un mélange eauméthanol $(50 \%-50 \%)$ avec une concentration de $1,5 \times 10^{-4} \mathrm{M} / 1$. Ceci permet un gain suffisant tout en gardant un rapport $\mathrm{d} n / \mathrm{d} T$ pour l'indice de réfraction assez faible et une capacité calorifique suffisante grâce à l'eau, ce qui minimise les distorsions thermiques du trajet optique dans le tube de colorant.

Nous avons fait varier la longueur d'onde d'émission de 6100 à $5750 \AA$, avec une rotation du filtre de 0 à $30^{\circ}$ environ, à l'aide d'une platine goniométrique commandée par une vis micrométrique permettant un étalonnage en longueur d'onde. La largeur spectrale d'émission est inférieure à $1 \AA$. Grâce au filtre spatial, la qualité optique du faisceau est excellente : faisceau gaussien de $4 \mathrm{~mm}$ de diamètre avec une divergence de $0,2 \mathrm{mrad}$, limitée en fait par la diffraction.

La cadence de répétition peut aller jusqu'à $20 \mathrm{~Hz}$, mais l'on note actuellement une baisse de $50 \%$ du rendement lorsque l'on passe de 5 à $20 \mathrm{~Hz}$, ceci étant dû sans doute au mauvais fonctionnement de l'éclateur 
qui sera remplacé par un thyratron. Ce laser a déjà accumulé plus de 500000 tirs sans baisse de performances. La durée de vie du colorant est supérieure à 350000 tirs pour 11 de Rh. $6 \mathrm{G}$, ce qui correspond à $100 \mathrm{~h}$ de fonctionnement à $10 \mathrm{~Hz}$, en raison du bon filtrage des rayons UV du flash par le réflecteur pyrex et par l'utilisation d'une couche réflectrice en argent qui diminue peut-être les performances de pompage par rapport à l'aluminium vers les basses longueurs d'onde, mais qui ne réfléchit pas les rayons UV en dessous de $3400 \AA$, responsables de la dégradation rapide du colorant.

4. Conclusion. - Nous avons réalisé un laser à colorant à cavité en anneau, pompé par flashs, de fonctionnement fiable et peu onéreux. Ce laser délivre des impulsions puissantes (quelques dizaines de $\mathrm{kW}$ ) à une cadence de $20 \mathrm{~Hz}$. L'accent a été mis surtout sur la simplicité de montage et la durée de fonctionnement des éléments, ainsi que sur la qualité spatiale et spectrale de l'onde émise grâce à une cavité résonnante en anneau à 4 miroirs, non plane pour compenser l'astigmatisme, avec filtrage spatial intra-cavité et balayage en fréquence par simple rotation d'un filtre interférentiel interchangeable pour couvrir des bandes spectrales différentes.

En montant le miroir $\mathrm{M}_{3}$ sur une céramique piézoélectrique commandée par un dispositif électronique en cours de réalisation, nous allons pouvoir asservir la longueur de la cavité interférométrique du laser à une valeur déterminée $(m \lambda / 2$ par exemple avec $m$ entier), de façon à réaliser l'amplification d'un laser continu monofréquence de longueur d'onde $\lambda$ par injection dans la cavité asservie en longueur, ainsi accordée sur la fréquence injectée. Le gain attendu sera alors de $10^{6}$ environ en puissance avec un très faible élargissement de la raie continue injectée. La construction d'un second laser identique nous donnera ainsi deux sources lumineuses puissantes et de grande finesse spectrale, permettant de réaliser des expériences de spectroscopie non linéaire à très haute résolution.

\section{Bibliographie}

[1] Pinard, J. et Liberman, S., Opt. Commun. 20 (1977) 344.

[2] Trehin, F., Grynberg, G. et Cagnac, B., Revue Phys. Appl. 13 (1978) 307

[3] Blit, S., Gagniel, U. and Treves, D., Appl. Phys. 12 (1977) 69.

[4] Hirth, A., Lasser, Th., Meyer, R. and Schetter, K., Opt. Commun. 34 (1980) 223.
[5] Chu, S. and Commins, E., Appl. Opt. 16 (1977) 2619.

[6] Jethwa, J. and Schafer, F. P., Appl. Phys. 4 (1974) 299.

[7] Chu, S. and Smith, R. W., Opt. Commun. 28 (1979) 2.

[8] Yee, T. K., Fan, B. and Gustafson, T. K., Appl. Opt. 18 (1979) 8 .

[9] Trehin, F., Thèse Docteur-Ingénieur, Paris (1979).

[10] KogelniK, H. and LI, T., Appl. Opt. 5 (1966) 10. 